\title{
An Introduction to BoSS-21 and a Framework to Build 3D Surface Human Models Using Anthropometric Constrains
}

\author{
Shi YIN, Rachida AMJOUN, Ali AVANNAKI \\ VisImage Systems Inc., Toronto, Canada
}

\begin{abstract}
This paper introduces a Body Sizing System for $21^{\text {st }}$ century (BoSS-21), which is a non-contact and automatic system to provide anthropometric measurement of an adult individual. BoSS-21 has been used in Canadian Military since 1999 for uniform design and purchase, anthropometric screening of aircraft pilot and vehicle drivers, as well as a general anthropometric survey.

This paper also introduces a framework to build a 3D surface human model using anthropometric constrains. This approach will generate a model, which is more anthropocentrically accurate comparing to the real human subject. To evaluate a $3 \mathrm{D}$ anthropometric model, we propose to use the differences on the anthropometric measures, such as defined in ISO7250, between the real subject and model under evaluation.
\end{abstract}

Keywords: BoSS-21, Anthropometry, 3D reconstruction, segmentation, deformation,

\section{Introduction}

Human bodies exist in a 3D space in nature. Although conventional anthropometry uses a set of anthropometric measures to describe human bodies, which are real numbers with zero degree of freedom, the definition of these measures are in 3D, such as defined in ISO7250 [1]. On the other hand, a $3 \mathrm{D}$ surface model of a human body can be used to extract the anthropometric information such as stature, acromial breath, chest circumference, and so on.

However an anthropometric model is not equivalent to a 3D surface human model. In addition to the anthropometric aspect, a 3D surface model of a human being can be useful for the purposes of visual art and human anatomy studies. Modern anthropometry is more related with biometrics, while human anatomy is more related with embryology.

For the purpose of engineering anthropometry and automatic data collection, Vislmage developed BoSS-21 (Sizing System for $21^{\text {st }}$ century) in later 90s. BoSS-21 has been used in Canadian military for over a decade in the applications of garment design/purchase, aircraft pilot screening, armed vehicle accommodation, and generic anthropometric survey.

Motivated by the application for anthropometric screening and visual presentation, we recently developed a framework to build a 3D surface human model using anthropometric constrains. We propose to optimize such a model to minimize the differences of the anthropometric measures between the generated 3D model and the real human being, Therefore, such a 3D model will be more anthropometrically accurate.

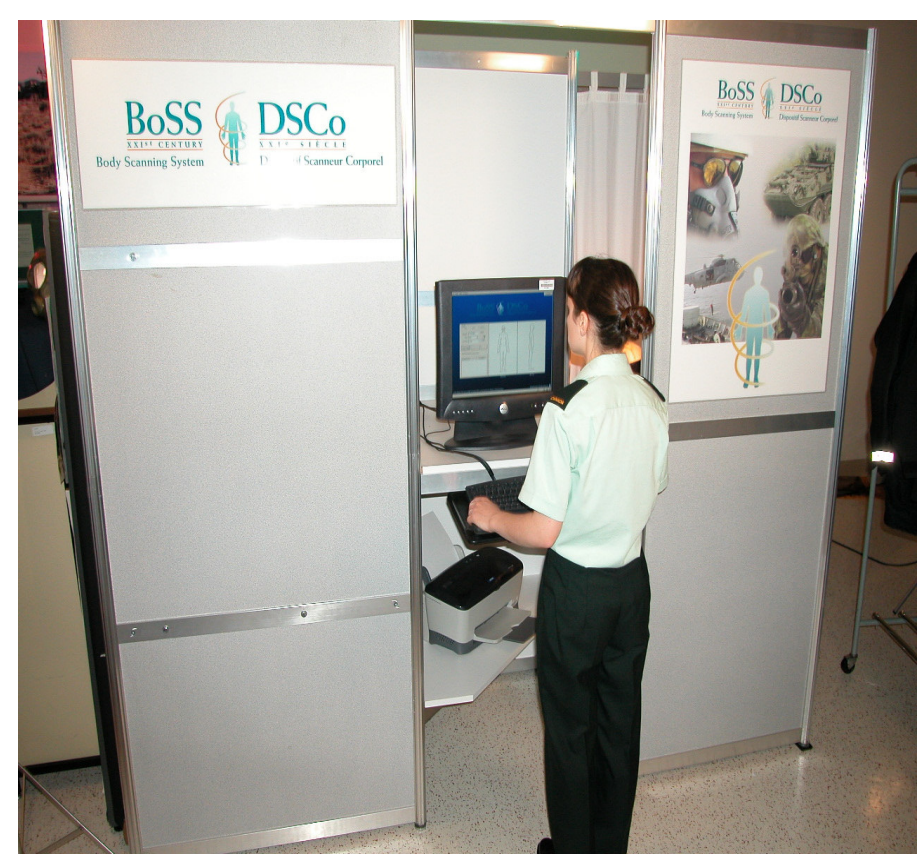

Figure 1: A Boss-21 system in operation 


\section{BoSS-21 and its applications}

BoSS-21 is a PC-based system comprised of two digital cameras and a blue backdrop embedded with calibration markers. The system takes simultaneous front and side pictures of individuals standing on the imaging platform. By taking both images simultaneously, the exact posture in space is captured, and it is possible to recover the object's three-dimensional size. A BoSS-21 system is shown in Figure 1.

BoSS-21 mobile edition has the same features as the standard edition, and only weight less than $40 \mathrm{~kg}$ and can be stored in a compact package as shown in figure 2. This edition is easy in transportation and simple to set-up.

BoSS-21 has been used in garment design and aircraft pilot screening using the anthropometric measures. A feature for the development of size allocation of a particular garment is shown in figure 3. One can use this feature to determine if/where additional sizes should be added and/or removed. Figure 4 shows an application for pilot screening, which has been used to assist the enrolment of pilot students.

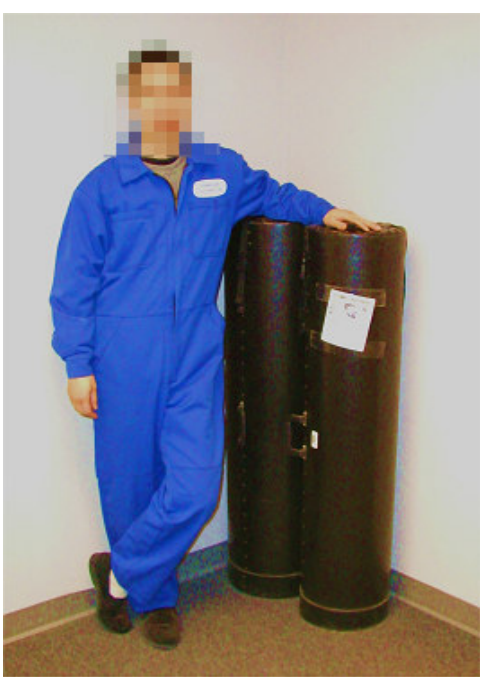

Figure 2: BoSS-21 Mobile Edition system

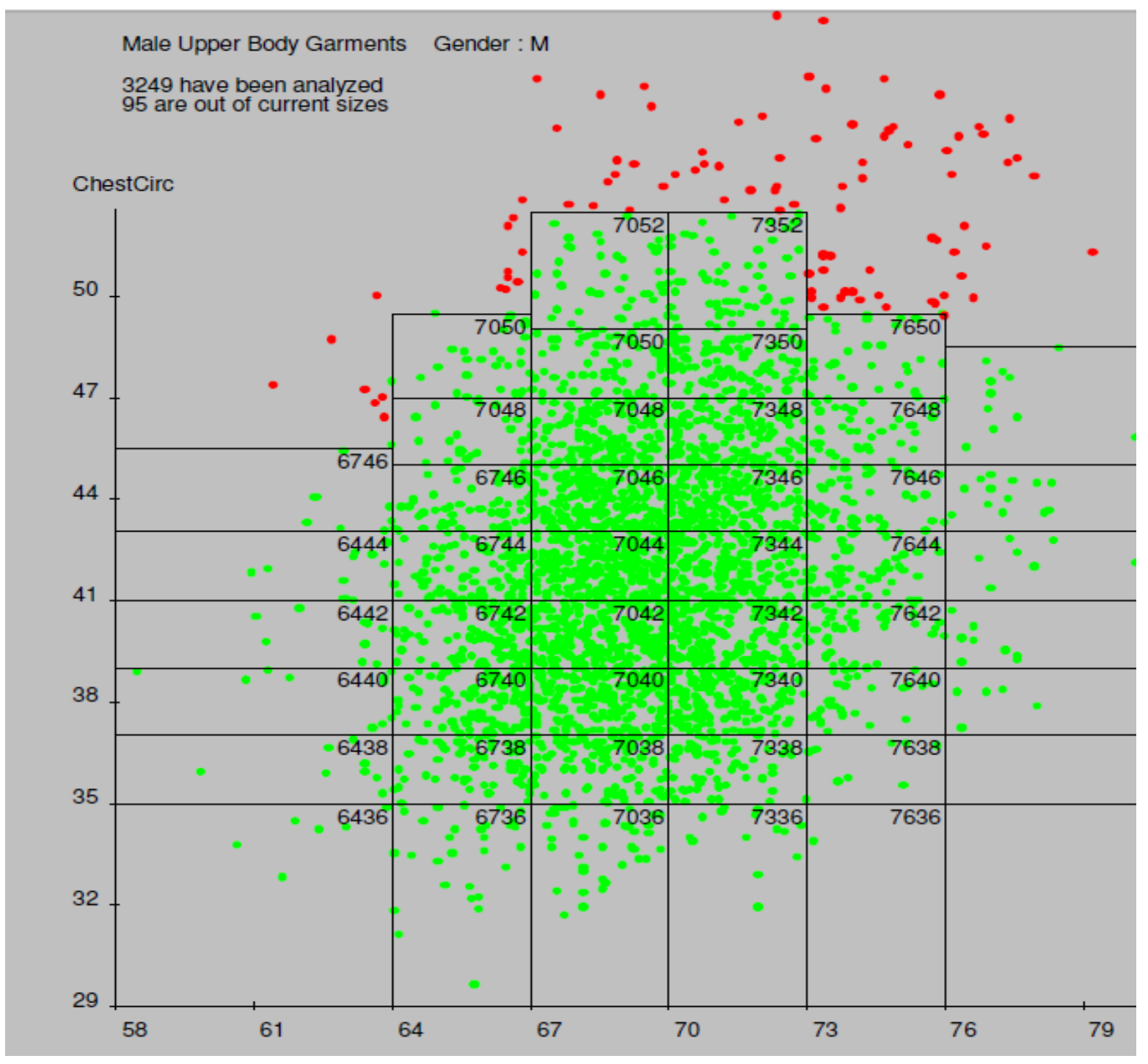

Figure 3: The anthropometric measures of a population in relation with a garment size allocation 


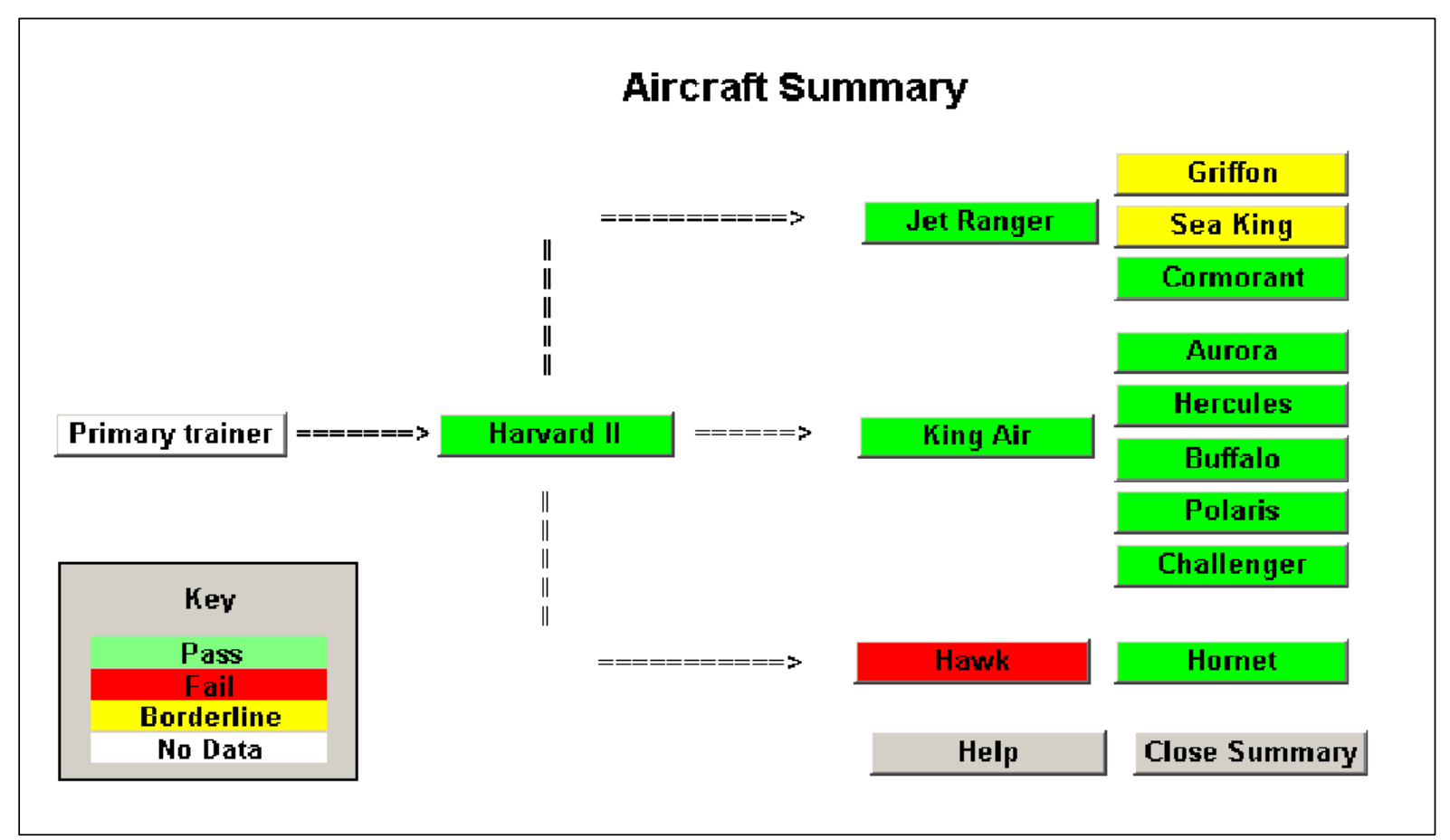

Figure 4: An output of the pilot anthropometric screening for an individual

\section{A framework to build a 3D surface human model}

Existing approaches used in certain 3D scanning systems generate 3D point cloud. This type geometric data maybe noisy, incomplete and may need large amount of data process for 3D surface model reconstruction. Due to the complexity of the reconstruction process, the error analysis of the reconstructed model is very critical.

We propose a new 3D surface human modeling framework that uses the anthropometric measures as constrains in the reconstruction and error evaluation processes. Given anthropometric measures of a human subject, his/her personal information and the generic model based on the general population, the proposed framework reconstructs 3D surface human model by adjusting the surface of the generic model by minimizing the anthropometric measures errors.

Our anthropometric measure-driven 3D surface human modeling has two main phases. The first phase extracts the predefined landmarks on the generic model and partitions the model into separated main body parts. The second phase uses the 3D landmarks detected on the real human subject from BoSS-21 to build the 3D surface model for this subject, by adjusting the surface of the generic model. The first is a pre-process phase, which needs to run once for any given generic model. The second is a running-time phase, which will run for each subject to be model. Figure 5 shows different steps within both phases in our system architecture.

\section{a. Pre-process Phase}

The 3D Generic model used in our approach is a 3D surface model in mesh representation. It contains geometry information and connectivity information. The geometry is described by a list of floating points that describe the positions of vertices. The connectivity is described by a list of integers that describe how these vertices are connected. We assume that the connectivity of the model remains constant, shared by all reconstructed models, and only the geometry varies, according to the input parameters. The Pre-process phase consists of two steps:

Landmarks extraction: In this step, a number of landmarks are extracted from the generic model. These predefined landmarks should highly correspond to the input landmarks generated from BoSS-21. Later, during the reconstruction process, these landmarks maybe adjusted to meet anthropometric constrains and to minimize the anthropometric measures errors. Figure 6 shows an example of the predefined landmark on the 3D generic model. 


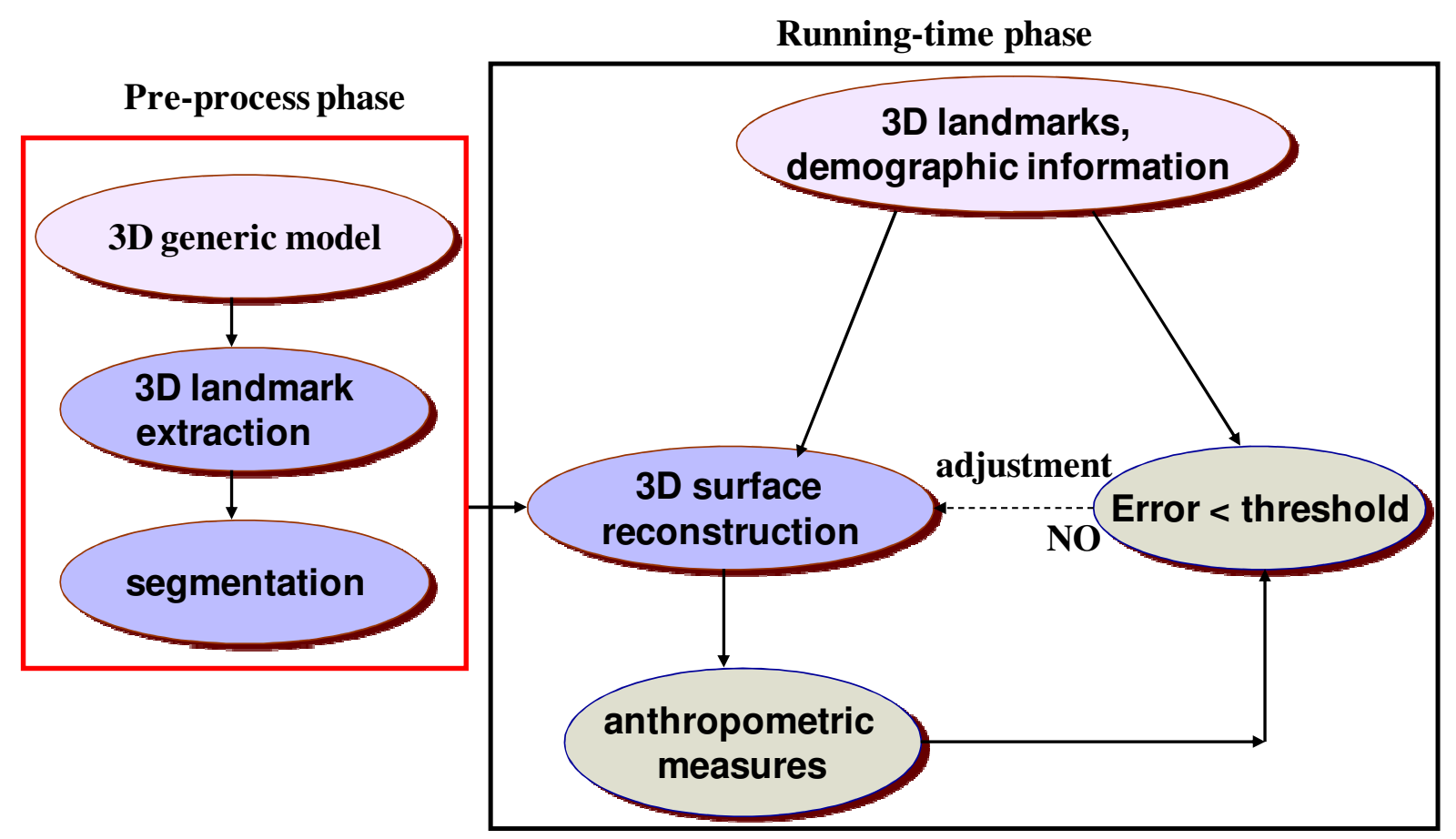

Figure 5: 3-D reconstruction system

Landmarks extraction: In this step, a number of landmarks are extracted from the generic model. These predefined landmarks should highly correspond to the input landmarks generated from BoSS-21. Later, during the reconstruction process, these landmarks maybe adjusted to meet anthropometric constrains and to minimize the anthropometric measures errors. Figure 6 shows an example of the predefined landmark on the 3D generic model.

Body model segmentation: In our framework, the segmentation is used to break down the $3 \mathrm{D}$ model into main body parts, which has become a necessary tool in geometric modeling. The goal behind the segmentation is: on the one hand, to gather the vertices which undergo a similar variation during deformation, and on the other hand, to preserve the local shape features.

\section{b. Running-Time Phase}

This phase is an optimization process trying to reconstruct a surface model by aligning the landmarks on the generic model with the ones from the real subject. Based on anthropometric constrains, each vertex in a body segment maybe transformed into a new position, forming a new surface. To preserve the local as well as the global shape, local coordinate frame is introduced in each segment before transformation.

After a new surface model is reconstructed, the accuracy of 3D human surface and the anthropometrical is evaluated by computing the error between the real subject and model under evaluation. If the error is over than predefined threshold, the surface is re-reconstructed, taking in consideration the anthropometric constrains. This process is iterated until, the differences of the anthropometric measures is minimized.

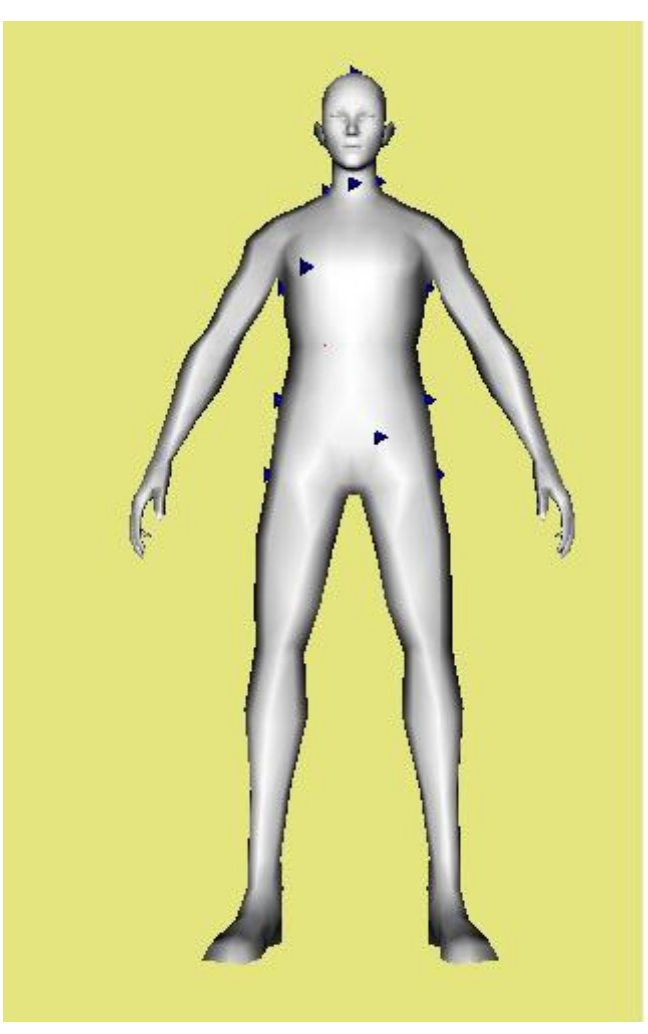

Figure 6: Example of 3D generic model 


\section{Conclusion}

In this paper, we introduced a Body Sizing System for $21^{\text {st }}$ century (BoSS-21), an automatic system to provide anthropometric measures of an adult individual. We also proposed a framework to generate a 3D surface model of human being. Our approach takes anthropometric data as constrains in the process to build 3D surface human model. Therefore the model built is more anthropocentrically accurate comparing to the real human subject.

\section{Acknowledgement}

The work on the development of framework to build a 3D surface human model using anthropometric constrains has been partially supported by the Industrial Research assistance Program (IRAP), the National Research Council of Canada (NRC). IRAP project \#742585.

\section{References}

1. ISO 7250 International Standard: Basic human body measurements for technical design, 1996 